\title{
A Study on $(E, 1)(E, q)$ Product Summability of Fourier Series and Its Conjugate Series
}

\author{
Kalpana Saxena ${ }^{1}$ \\ ${ }^{1}$ Department of Mathematics, \\ Govt. M.V.M. Barkatullah University \\ Bhopal, India
}

\author{
Sheela Verma ${ }^{2}$ \\ Department of Mathematics, \\ Govt. M.V.M. Barkatullah University \\ Bhopal, India
}

\begin{abstract}
In this paper, introduce the concept of $(E, 1)(E, q)$ product operators and establishes two new theorems on $(E, 1)(E, q)$ product Summability of Fourier series and its conjugate series. The results obtained in the paper further extend several known result on linear operators.
\end{abstract}

Keyword: (E,q) Summability, (E,1) Summability, (E,1) (E,q) Summability.

\section{INTRODUCTION}

In this field of Summability of Fourier series \& its allied series, the product Summability $(\mathrm{E}, \mathrm{q})(\mathrm{X}),(\mathrm{X})(\mathrm{E}, \mathrm{q})$ or $|\mathrm{E}, \mathrm{q}|$ have be studied by a number of researchers like, Mohanty,R. and Mohapatra, S.(1968), Kwee, B.(19722), ${ }^{2}$ chandra, P.(1977), ${ }^{1}$ chandra, P. and Dikshit, G.D.(1981), sachan,M.P.(1983), Bhagwat, Purnima(1987), Nigam, H.K. and Sharama, Ajay(2006), lal, S. Singh,H.P. Tiwari, ${ }^{8}$ Sandeep kumar, and Bariwal, Chandrashekhar (2010), ${ }^{3}$ Dhakal, Binod Prasad (2011), Rathore, H.L. and Shrivasstava, U.K. (2012), Nigam, H.K. and Sharma, K.(2012,2013), Sinha, Santosh Kumar and Shrivastava, U.K.(2014), Mishara,V.N. Sonavane, Vaishali(2015) and many more, under various type of criteria and conditions. After this, so many results established on double factorable Summability of double Fourier series, But nothing seems to have been done so far to study (E,1) (E,q) product Summability of Fourier series and its conjugates series. Therefore, in this paper, two theorems on (E,1) (E,q) Summability of Fourier series and its conjugate series have been proved under a general condition.

\section{DEFINition AND Notation}

Let $f(x)$ be a $2 \pi$ - periodic function and Lebesgue integrable over $(-\pi, \pi)$. The Fourier series of $f(x)$ is given by

$$
\mathrm{f}(\mathrm{x}) \sim \frac{\mathrm{a}_{0}}{2}+\sum_{\mathrm{n}=1}^{\infty}\left(\mathrm{a}_{\mathrm{n}} \cos \mathrm{nx}+\mathrm{b}_{\mathrm{n}} \sin \mathrm{nx}\right) \equiv \sum_{\mathrm{n}=1}^{\infty} \mathrm{A}_{\mathrm{n}}(\mathrm{x})
$$

The conjugate series of Fourier series is given by

$$
\sum_{n=1}^{\infty}\left(b_{n} \cos n x-a_{n} \sin n x\right) \equiv \sum_{n=1}^{\infty} B_{n}(x)
$$

We shall use the following notations:

$$
\begin{gathered}
\Phi(\mathrm{t})=\mathrm{f}(\mathrm{x}+\mathrm{t})+\mathrm{f}(\mathrm{x}-\mathrm{t})-2 \mathrm{~S} \\
\psi(\mathrm{t})=\mathrm{f}(\mathrm{x}+\mathrm{t})-\mathrm{f}(\mathrm{x}-\mathrm{t}) \\
\mathrm{K}_{\mathrm{n}}(\mathrm{t})=\frac{1}{2^{\mathrm{n}+1} \cdot \pi} \sum_{\mathrm{k}=0}^{\mathrm{n}}\left[\frac{1}{(1+\mathrm{q})^{\mathrm{k}}}\left(\begin{array}{l}
\mathrm{n} \\
\mathrm{k}
\end{array}\right)\left\{\sum_{\mathrm{v}=0}^{\mathrm{k}}\left(\begin{array}{l}
\mathrm{k} \\
\mathrm{v}
\end{array}\right) \mathrm{q}^{\mathrm{k}-\mathrm{v}} \frac{\sin \left(\mathrm{v}+\frac{1}{2}\right) \mathrm{t}}{\sin \frac{\mathrm{t}}{2}}\right\}\right] \\
\widetilde{\mathrm{K}_{\mathrm{n}}}(\mathrm{t})=\frac{1}{2^{\mathrm{n}+1} \cdot \pi} \sum_{\mathrm{k}=0}^{\mathrm{n}}\left[\frac{1}{(1+\mathrm{q})^{\mathrm{k}}}\left(\begin{array}{l}
\mathrm{n} \\
\mathrm{k}
\end{array}\right)\left\{\sum_{\mathrm{v}=0}^{\mathrm{k}}\left(\begin{array}{l}
\mathrm{k} \\
\mathrm{v}
\end{array}\right) \mathrm{q}^{\mathrm{k}-\mathrm{v}} \frac{\cos \left(\mathrm{v}+\frac{1}{2}\right) \mathrm{t}}{\sin \frac{\mathrm{t}}{2}}\right\}\right]
\end{gathered}
$$


And $\tau=\left[\frac{1}{\mathrm{t}}\right]$, where $\tau$ denotes the greatest integer not greater than $\frac{1}{\mathrm{t}}$

Let $\sum_{n=0}^{\infty} u_{n}$ be a given infinite series with sequence of its $n^{\text {th }}$ partial some sum of $\left\{S_{n}\right\}$. The $(E, 1)$ transform is defined as the $\mathrm{n}^{\text {th }}$ partial sum of $(\mathrm{E}, 1)$ Summability and is given by

$$
\mathrm{E}_{\mathrm{n}}^{1}=\mathrm{t}_{\mathrm{n}}=\frac{1}{2^{\mathrm{n}}} \sum_{\mathrm{k}=0}^{\mathrm{n}}\left(\begin{array}{l}
\mathrm{n} \\
\mathrm{k}
\end{array}\right) \mathrm{s}_{\mathrm{k}} \rightarrow \mathrm{s} \text { as } \mathrm{n} \rightarrow \infty
$$

then the infinite series $\sum_{\mathrm{n}=0}^{\infty} \mathrm{u}_{\mathrm{n}}$ is summable to the definite number s by (E,q) method.

If,

$$
(E, q)=E_{k}^{\mathrm{q}}=\frac{1}{(1+q)^{k}} \sum_{v=0}^{\mathrm{k}}\left(\begin{array}{l}
\mathrm{k} \\
\mathrm{v}
\end{array}\right) \mathrm{q}^{\mathrm{k}-\mathrm{v}} \mathrm{s}_{\mathrm{v}} \rightarrow \mathrm{s} \text { as } \mathrm{n} \rightarrow \infty
$$

then the infinite series $\sum_{n=0}^{\infty} u_{n}$ is summable to the definite no. $s$ by $(E, 1)(E, q)$ Summability method. If,

$$
\mathrm{E}_{\mathrm{n}}^{1} \mathrm{E}_{\mathrm{k}}^{\mathrm{q}}=\mathrm{t}_{\mathrm{n}}^{(\mathrm{E}, 1)(\mathrm{E}, \mathrm{q})}=\frac{1}{2^{\mathrm{n}}} \sum_{\mathrm{k}=0}^{\mathrm{n}}\left(\begin{array}{l}
\mathrm{n} \\
\mathrm{k}
\end{array}\right) \mathrm{E}_{\mathrm{k}}^{\mathrm{q}} \rightarrow \mathrm{s} \text {, as } \mathrm{n} \rightarrow \infty
$$

\section{MAIn TheOREMS}

We prove the following theorems,

3.1 Theorem. Let $\left\{p_{n}\right\}$ be a positive, monotonic, non-increasing sequence of real constants such that

$$
\mathrm{p}_{\mathrm{n}}=\sum_{\mathrm{v}=0}^{\mathrm{n}} \mathrm{p}_{\mathrm{v}} \rightarrow \infty \text { as } \mathrm{n} \rightarrow \infty
$$

if,

$$
\varnothing(\mathrm{t})=\int_{0}^{\mathrm{t}}|\varnothing(\mathrm{u})| \mathrm{du}=\mathrm{o}\left[\frac{\mathrm{t}}{\alpha\left(\frac{1}{\mathrm{t}}\right), \mathrm{p}_{\mathrm{t}}}\right] \text {, as } \mathrm{t} \rightarrow+0
$$

Where, $\alpha(\mathrm{t})$ is positive, monotonic and non-increasing function of $\mathrm{t}$ and

$$
\log n=O\left[\{\alpha(n)\} \cdot p_{n}\right] \text {, as } n \rightarrow \infty
$$

Then the Fourier series (2.1) is summable (E,1)(E,q) to $f(x)$.

3.2 Theorem. Let $\left\{p_{n}\right\}$ be a positive, monotonic, non-increasing sequence of real constants such that

$$
\mathrm{p}_{\mathrm{n}}=\sum_{\mathrm{v}=0}^{\mathrm{n}} \mathrm{p}_{\mathrm{v}} \rightarrow \infty \text { as } \mathrm{n} \rightarrow \infty
$$

If,

$$
\psi(\mathrm{t})=\int_{0}^{\mathrm{t}}|\psi(\mathrm{u})| \mathrm{du}=\mathrm{o}\left[\frac{\mathrm{t}}{\alpha\left(\frac{1}{\mathrm{t}}\right), \mathrm{p}_{\mathrm{t}}}\right], \text { as } \mathrm{t} \rightarrow+0
$$

where $\alpha(t)$ is a positive, monotonic and non-increasing function of $t$, then the conjugate Fourier series (2.2) is summable to $(\mathrm{E}, 1)(\mathrm{E}, \mathrm{q})$ to

$$
\tilde{\mathrm{f}}(\mathrm{x})=\frac{-1}{2 \pi} \int_{0}^{2 \pi} \psi(\mathrm{t}) \cot \left(\frac{\mathrm{t}}{2}\right) \mathrm{dt}
$$

at any point where this point exists.

\section{LEMMAS}

Lemma 1. $\left|k_{n}(t)\right|=O(n)$, for $0 \leq t \leq \frac{1}{n} ; \sin n t \leq n \sin n t ;|\cos n t \leq 1|$

$$
\text { Proof: }\left|\mathrm{k}_{\mathrm{n}}(\mathrm{t})\right| \leq \frac{1}{2^{\mathrm{n}+1} \cdot \pi}\left|\sum_{\mathrm{k}=0}^{\mathrm{n}}\left[\frac{1}{(1+\mathrm{q})^{\mathrm{k}}}\left(\begin{array}{l}
\mathrm{n} \\
\mathrm{k}
\end{array}\right) \sum_{\mathrm{v}=0}^{\mathrm{k}}\left(\begin{array}{l}
\mathrm{k} \\
\mathrm{v}
\end{array}\right) \mathrm{q}^{\mathrm{k}-\mathrm{v}} \frac{\sin \left(\mathrm{v}+\frac{1}{2}\right) \mathrm{t}}{\sin \frac{\mathrm{t}}{2}}\right]\right|
$$




$$
\begin{gathered}
\leq \frac{1}{2^{\mathrm{n}+1} \cdot \pi}\left|\sum_{\mathrm{k}=0}^{\mathrm{n}}\left[\frac{1}{(1+\mathrm{q})^{\mathrm{k}}}\left(\begin{array}{l}
\mathrm{n} \\
\mathrm{k}
\end{array}\right) \sum_{\mathrm{v}=0}^{\mathrm{k}}\left(\begin{array}{l}
\mathrm{k} \\
\mathrm{v}
\end{array}\right) \mathrm{q}^{\mathrm{k}-\mathrm{v}} \frac{\left.(2 \mathrm{v}+1) \sin \frac{\mathrm{t}}{2}\right]}{\sin \frac{\mathrm{t}}{2}}\right]\right| \\
\leq \frac{1}{2^{\mathrm{n}+1} \cdot \pi}\left|\sum_{\mathrm{k}=0}^{\mathrm{n}}\left[\frac{1}{(1+\mathrm{q})^{\mathrm{k}}}\left(\begin{array}{l}
\mathrm{n} \\
\mathrm{k}
\end{array}\right)(2 \mathrm{k}+1) \sum_{\mathrm{v}=0}^{\mathrm{k}}\left(\begin{array}{l}
\mathrm{k} \\
\mathrm{v}
\end{array}\right) \mathrm{q}^{\mathrm{k}-\mathrm{v}}\right]\right| \\
=\frac{1}{2^{\mathrm{n}+1} \cdot \pi} \sum_{\mathrm{k}=0}^{\mathrm{n}}\left(\begin{array}{l}
\mathrm{n} \\
\mathrm{k}
\end{array}\right)(2 \mathrm{k}+1) \\
=\frac{1}{2^{\mathrm{n}+1} \cdot \pi}(2 \mathrm{n}+1) \sum_{\mathrm{k}=0}^{\mathrm{n}}\left(\begin{array}{l}
\mathrm{n} \\
\mathrm{k}
\end{array}\right) \\
=\frac{2 \mathrm{n}+1}{2 \pi} \\
=0(\mathrm{n})
\end{gathered}
$$

Lemma $2\left|k_{n}(t)\right|=o\left(\frac{1}{t}\right)$, for $\frac{1}{n} \leq t \leq \pi ; \sin (t / 2) \geq t / 2$ and $\sin n t \leq 1$

$$
\begin{gathered}
\left|k_{n}(t)\right| \leq \frac{1}{2^{n+1} \cdot \pi}\left|\sum_{k=0}^{n}\left[\frac{1}{(1+q)^{k}}\left(\begin{array}{l}
n \\
k
\end{array}\right) \sum_{v=0}^{k}\left(\begin{array}{l}
k \\
v
\end{array}\right) q^{k-v} \frac{\sin \left(v+\frac{1}{2}\right) t}{\sin \frac{t}{2}}\right]\right| \\
\leq \frac{1}{2^{n+1} \cdot \pi}\left|\sum_{k=0}^{n}\left[\frac{1}{(1+q)^{k}}\left(\begin{array}{l}
n \\
k
\end{array}\right) \sum_{v=0}^{k}\left(\begin{array}{l}
k \\
v
\end{array}\right) q^{k-v} \cdot \frac{1}{t / 2}\right]\right| \\
=\frac{1}{2^{n} \cdot \pi t}\left[\sum_{k=0}^{n}\left(\begin{array}{l}
n \\
k
\end{array}\right) \sum_{v=0}^{k}\left(\begin{array}{l}
k \\
v
\end{array}\right)\right] \\
=\frac{1}{2^{n} \cdot \pi t}\left[\sum_{k=0}^{n}\left(\begin{array}{l}
n \\
k
\end{array}\right)\right] \\
=\frac{1}{\pi t} \\
=0\left(\frac{1}{t}\right)
\end{gathered}
$$

Lemma 3. $\widetilde{k_{n}}(t)=0\left(\frac{1}{t}\right)$, for $0 \leq t \leq \frac{1}{n} ; \sin (t / 2) \geq t / 2 ;|\cos n t| \leq 1$

$$
\begin{gathered}
\left|\widetilde{\mathrm{k}_{\mathrm{n}}}(\mathrm{t})\right| \leq \frac{1}{2^{\mathrm{n}+1} \cdot \pi}\left|\sum_{\mathrm{k}=0}^{\mathrm{n}}\left[\frac{1}{(1+\mathrm{q})^{\mathrm{k}}}\left(\begin{array}{l}
\mathrm{n} \\
\mathrm{k}
\end{array}\right) \sum_{\mathrm{v}=0}^{\mathrm{k}}\left(\begin{array}{l}
\mathrm{k} \\
\mathrm{v}
\end{array}\right) \mathrm{q}^{\mathrm{k}-\mathrm{v}} \frac{\cos \left(\mathrm{v}+\frac{1}{2}\right) \mathrm{t}}{\sin \frac{\mathrm{t}}{2}}\right]\right| \\
\leq \frac{1}{2^{\mathrm{n}+1} \cdot \pi} \sum_{\mathrm{k}=0}^{\mathrm{n}}\left[\frac{1}{(1+\mathrm{q})^{\mathrm{k}}}\left(\begin{array}{l}
\mathrm{n} \\
\mathrm{k}
\end{array}\right) \sum_{\mathrm{v}=0}^{\mathrm{k}}\left(\begin{array}{l}
\mathrm{k} \\
\mathrm{v}
\end{array}\right) \mathrm{q}^{\mathrm{k}-\mathrm{v}}\left|\frac{\cos \left(\mathrm{v}+\frac{1}{2}\right) \mathrm{t}}{\sin \mathrm{t} / 2}\right|\right] \\
\leq \frac{1}{2^{\mathrm{n}} \cdot \pi \mathrm{t}} \sum_{\mathrm{k}=0}^{\mathrm{n}}\left[\frac{1}{(1+\mathrm{q})^{\mathrm{k}}}\left(\begin{array}{l}
\mathrm{n} \\
\mathrm{k}
\end{array}\right) \sum_{\mathrm{v}=0}^{\mathrm{k}}\left(\begin{array}{l}
\mathrm{k} \\
\mathrm{v}
\end{array}\right) \mathrm{q}^{\mathrm{k}-\mathrm{v}}\right] \\
=\frac{1}{2^{\mathrm{n}} \cdot \pi \mathrm{t}}\left[\sum_{\mathrm{k}=0}^{\mathrm{n}}\left(\begin{array}{l}
\mathrm{n} \\
\mathrm{k}
\end{array}\right)\right]
\end{gathered}
$$




$$
\begin{gathered}
=\frac{1}{\pi \mathrm{t}} \\
=0\left(\frac{1}{\mathrm{t}}\right)
\end{gathered}
$$

Lemmas 4. $\left|\widetilde{\mathrm{k}_{n}}(\mathrm{t})\right|=0\left(\frac{1}{\mathrm{t}}\right)$, for $\frac{1}{\mathrm{n}} \leq \mathrm{t} \leq \pi, \sin (\mathrm{t} / 2) \geq \mathrm{t} / 2$

$$
\begin{aligned}
& \text { Proof:- }\left|\widetilde{\mathrm{k}_{\mathrm{n}}}(\mathrm{t})\right| \leq \frac{1}{2^{\mathrm{n}+1} \cdot \pi}\left|\sum_{\mathrm{k}=0}^{\mathrm{n}}\left[\frac{1}{(1+\mathrm{q})^{\mathrm{k}}}\left(\begin{array}{l}
\mathrm{n} \\
\mathrm{k}
\end{array}\right) \sum_{\mathrm{v}=0}^{\mathrm{k}}\left(\begin{array}{l}
\mathrm{k} \\
\mathrm{v}
\end{array}\right) \mathrm{q}^{\mathrm{k}-\mathrm{v}} \frac{\cos \left(\mathrm{v}+\frac{1}{2}\right) \mathrm{t}}{\sin \frac{\mathrm{t}}{2}}\right]\right| \\
& \leq \frac{1}{2^{\mathrm{n}} \cdot \pi \mathrm{t}}\left|\sum_{\mathrm{k}=0}^{\mathrm{n}}\left[\frac{1}{(1+\mathrm{q})^{\mathrm{k}}}\left(\begin{array}{l}
\mathrm{n} \\
\mathrm{k}
\end{array}\right) \operatorname{Re}\left\{\sum_{\mathrm{v}=0}^{\mathrm{k}}\left(\begin{array}{l}
\mathrm{k} \\
\mathrm{v}
\end{array}\right) \mathrm{q}^{\mathrm{k}-\mathrm{v}} \mathrm{e}^{\mathrm{i}\left(\mathrm{v}+\frac{1}{2}\right) \mathrm{t}}\right\}\right]\right| \\
& \leq \frac{1}{2^{\mathrm{n}} \cdot \pi \mathrm{t}}\left|\sum_{\mathrm{k}=0}^{\mathrm{n}}\left[\frac{1}{(1+\mathrm{q})^{\mathrm{k}}}\left(\begin{array}{l}
\mathrm{n} \\
\mathrm{k}
\end{array}\right) \operatorname{Re}\left\{\sum_{\mathrm{v}=0}^{\mathrm{k}}\left(\begin{array}{l}
\mathrm{k} \\
\mathrm{v}
\end{array}\right) \mathrm{q}^{\mathrm{k}-\mathrm{v}} \mathrm{e}^{\mathrm{ivt}}\right\}\right]\right|\left|\mathrm{e}^{\mathrm{it} / 2}\right| \\
& \leq \frac{1}{2^{\mathrm{n}} \cdot \pi \mathrm{t}}\left|\sum_{\mathrm{k}=0}^{\mathrm{n}}\left[\frac{1}{(1+\mathrm{q})^{\mathrm{k}}}\left(\begin{array}{l}
\mathrm{n} \\
\mathrm{k}
\end{array}\right) \operatorname{Re}\left\{\sum_{\mathrm{v}=0}^{\mathrm{k}}\left(\begin{array}{l}
\mathrm{k} \\
\mathrm{v}
\end{array}\right) \mathrm{q}^{\mathrm{k}-\mathrm{v}} \mathrm{e}^{\mathrm{ivt}}\right\}\right]\right| \\
& \leq \frac{1}{2^{\mathrm{n}} \cdot \pi \mathrm{t}}\left|\sum_{\mathrm{k}=0}^{\tau-1}\left[\frac{1}{(1+\mathrm{q})^{\mathrm{k}}}\left(\begin{array}{l}
\mathrm{n} \\
\mathrm{k}
\end{array}\right) \operatorname{Re}\left\{\sum_{\mathrm{v}=0}^{\mathrm{k}}\left(\begin{array}{l}
\mathrm{k} \\
\mathrm{v}
\end{array}\right) \mathrm{q}^{\mathrm{k}-\mathrm{v}} \mathrm{e}^{\mathrm{ivt}}\right\}\right]\right|+ \\
& \frac{1}{2^{\mathrm{n}} \cdot \pi \mathrm{t}}\left|\sum_{\mathrm{k}=\tau}^{\mathrm{n}}\left[\frac{1}{(1+\mathrm{q})^{\mathrm{k}}}\left(\begin{array}{l}
\mathrm{n} \\
\mathrm{k}
\end{array}\right) \operatorname{Re}\left\{\sum_{\mathrm{v}=0}^{\mathrm{k}}\left(\begin{array}{l}
\mathrm{k} \\
\mathrm{v}
\end{array}\right) \mathrm{q}^{\mathrm{k}-\mathrm{v}} \mathrm{e}^{\mathrm{ivt}}\right\}\right]\right| \\
& =\mathrm{k}_{1}+\mathrm{k}_{2}
\end{aligned}
$$

Now,

$$
\begin{gathered}
\left|\mathrm{k}_{1}\right| \leq \frac{1}{2^{\mathrm{n}} \cdot \pi \mathrm{t}}\left|\sum_{\mathrm{k}=0}^{\tau-1}\left[\frac{1}{(1+\mathrm{q})^{\mathrm{k}}}\left(\begin{array}{l}
\mathrm{n} \\
\mathrm{k}
\end{array}\right) \operatorname{Re}\left\{\sum_{\mathrm{v}=0}^{\mathrm{k}}\left(\begin{array}{l}
\mathrm{k} \\
\mathrm{v}
\end{array}\right) \mathrm{q}^{\mathrm{k}-\mathrm{v}} \mathrm{e}^{\mathrm{ivt}}\right\}\right]\right| \\
\leq \frac{1}{2^{\mathrm{n}} \cdot \pi \mathrm{t}}\left|\sum_{\mathrm{k}=0}^{\tau-1}\left[\frac{1}{(1+\mathrm{q})^{\mathrm{k}}}\left(\begin{array}{l}
\mathrm{n} \\
\mathrm{k}
\end{array}\right) \sum_{\mathrm{v}=0}^{\mathrm{k}}\left(\begin{array}{l}
\mathrm{k} \\
\mathrm{v}
\end{array}\right) \mathrm{q}^{\mathrm{k}-\mathrm{v}}\right]\right| \mathrm{e}^{\mathrm{ivt}} \mid \\
\leq \frac{1}{2^{\mathrm{n}} \cdot \pi \mathrm{t}}\left|\sum_{\mathrm{k}=0}^{\tau-1} \frac{1}{\left(1+\mathrm{q}^{\mathrm{k}}\right)}\left(\begin{array}{l}
\mathrm{n} \\
\mathrm{k}
\end{array}\right) \sum_{\mathrm{v}=0}^{\mathrm{k}}\left(\begin{array}{l}
\mathrm{k} \\
\mathrm{v}
\end{array}\right) \mathrm{q}^{\mathrm{k}-\mathrm{v}}\right| \\
\leq \frac{1}{2^{\mathrm{n}} \cdot \pi \mathrm{t}} \sum_{\mathrm{k}=0}^{\tau-1}\left(\begin{array}{l}
\mathrm{n} \\
\mathrm{k}
\end{array}\right) \\
=0\left(\frac{1}{\mathrm{t}}\right)
\end{gathered}
$$

And

$$
\left|k_{2}\right| \leq \frac{1}{2^{n} \cdot \pi t}\left|\sum_{k=\tau}^{n}\left[\frac{1}{(1+q)^{k}}\left(\begin{array}{l}
n \\
k
\end{array}\right) \operatorname{Re}\left\{\sum_{v=0}^{k}\left(\begin{array}{l}
k \\
v
\end{array}\right) q^{k-v} e^{i v t}\right\}\right]\right|
$$




$$
\begin{gathered}
\leq \frac{1}{2^{\mathrm{n}} \cdot \pi \mathrm{t}} \sum_{\mathrm{k}=\tau}^{\mathrm{n}} \frac{1}{(1+\mathrm{q})^{\mathrm{k}}}\left(\begin{array}{l}
\mathrm{n} \\
\mathrm{k}
\end{array}\right) \quad \max _{0} \leq \mathrm{m} \leq \mathrm{k}\left|\sum_{\mathrm{v}=0}^{\mathrm{k}}\left(\begin{array}{l}
\mathrm{k} \\
\mathrm{v}
\end{array}\right) \mathrm{q}^{\mathrm{k}-\mathrm{v}} \mathrm{e}^{\mathrm{ivt}}\right| \\
\leq \frac{1}{2^{\mathrm{n}} \cdot \pi \mathrm{t}}(1+\mathrm{q})^{\tau} \sum_{\mathrm{k}=\tau}^{\mathrm{n}} \frac{1}{(1+\mathrm{q})^{\mathrm{k}}}\left(\begin{array}{l}
\mathrm{n} \\
\mathrm{k}
\end{array}\right) \\
=\frac{1}{2^{\mathrm{n}} \cdot \pi \mathrm{t}} \sum_{\mathrm{k}=\tau}^{\mathrm{n}}\left(\begin{array}{l}
\mathrm{n} \\
\mathrm{k}
\end{array}\right) \\
=0\left(\frac{1}{\mathrm{t}}\right)
\end{gathered}
$$

\section{Proof of Main Theorems}

\subsection{Proof of theorem}

Following Titchmash [8] and using Riemann-Lebesgue theorem, $S_{n}(f ; x)$ of the series (2.1) is given by

$$
S_{n}(f ; x)-f(x)=\frac{1}{2 \pi} \int_{0}^{\pi} \emptyset(t) \frac{\sin \left(n+\frac{1}{2}\right) t}{\sin \frac{t}{2}} d t
$$

Therefore using (2.1), the (E,q), transform $E_{n}^{q}$ of $S_{n}(f ; x)$ is given by

$$
E_{n}^{q}-f(x)=\frac{1}{2 \pi(1+q)^{k}} \int_{0}^{\pi} \phi(t)\left\{\sum_{v=0}^{k}\left(\begin{array}{l}
k \\
v
\end{array}\right) q^{k-v} \frac{\sin \left(k+\frac{1}{2}\right) t}{\sin \frac{t}{2}}\right\} d t
$$

Now denoting $(E, 1)(E, q)$ transform of $S_{n}(f ; x)$ by $E_{n}^{1} E_{n}^{q}$ we write

$$
\begin{gathered}
\mathrm{E}_{\mathrm{n}}^{1} \mathrm{E}_{\mathrm{n}}^{\mathrm{q}}-\mathrm{f}(\mathrm{x})=\frac{1}{2 \pi \cdot(2)^{\mathrm{n}}} \sum_{\mathrm{k}=0}^{\mathrm{n}}\left[\frac{1}{(1+\mathrm{q})^{\mathrm{k}}}\left(\begin{array}{l}
\mathrm{n} \\
\mathrm{k}
\end{array}\right) \int_{0}^{\pi} \emptyset(\mathrm{t})\left\{\sum_{\mathrm{v}=0}^{\mathrm{k}}\left(\begin{array}{l}
\mathrm{k} \\
\mathrm{v}
\end{array}\right) \mathrm{q}^{\mathrm{k}-\mathrm{v}} \frac{\sin \left(\mathrm{k}+\frac{1}{2}\right) \mathrm{t}}{\sin \frac{\mathrm{t}}{2}}\right\} \mathrm{dt}\right] \\
=\frac{1}{2^{\mathrm{n}+1} \cdot \pi} \sum_{\mathrm{k}=0}^{\mathrm{n}}\left[\frac{1}{(1+\mathrm{q})^{\mathrm{k}}}\left(\begin{array}{l}
\mathrm{n} \\
\mathrm{k}
\end{array}\right) \int_{0}^{\pi} \emptyset(\mathrm{t})\left\{\sum_{\mathrm{v}=0}^{\mathrm{k}}\left(\begin{array}{l}
\mathrm{k} \\
\mathrm{v}
\end{array}\right) \mathrm{q}^{\mathrm{k}-\mathrm{v}} \frac{\sin \left(\mathrm{k}+\frac{1}{2}\right) \mathrm{t}}{\sin \frac{\mathrm{t}}{2}}\right\} \mathrm{dt}\right] \\
=\int_{0}^{\pi} \emptyset(\mathrm{t}) \mathrm{k}_{\mathrm{n}}(\mathrm{t}) \mathrm{dt}
\end{gathered}
$$

In order to prove the theorem, we have to show that, under the hypothesis of theorem

$$
\begin{aligned}
\int_{0}^{\pi} \emptyset(\mathrm{t}) \mathrm{k}_{\mathrm{n}}(\mathrm{t}) \mathrm{dt}=\mathrm{o}(1), \text { as } \mathrm{n} \rightarrow \infty \\
\text { For } 0<\delta<\pi, \text { We have } \\
\int_{0}^{\pi} \emptyset(\mathrm{t}) \mathrm{k}_{\mathrm{n}}(\mathrm{t}) \mathrm{dt}=\left[\int_{0}^{1 / \mathrm{n}} \emptyset(\mathrm{t})+\int_{1 / \mathrm{n}}^{\delta} \emptyset(\mathrm{t})+\int_{\delta}^{\pi} \emptyset(\mathrm{t})\right] \mathrm{k}_{\mathrm{n}}(\mathrm{t}) \mathrm{dt} \\
=\mathrm{I}_{1}+\mathrm{I}_{2}+\mathrm{I}_{3} \text { (say) }
\end{aligned}
$$

We consider,

$$
\begin{gathered}
\left|\mathrm{I}_{1}\right| \leq \int_{0}^{1 / \mathrm{n}}|\varnothing(\mathrm{t})|\left|\mathrm{k}_{\mathrm{n}}(\mathrm{t})\right| \mathrm{dt} \\
=0(\mathrm{n})\left[\int_{0}^{1 / \mathrm{n}}|\varnothing(\mathrm{t})| \mathrm{dt}\right] \text { by lemma } 1
\end{gathered}
$$




$$
\begin{aligned}
=O(n) & {\left[o\left\{\frac{1}{n \alpha(n) \cdot p_{n}}\right\}\right] \text { by (3.1) } } \\
= & o\left\{\frac{1}{\alpha(n) \cdot p_{n}}\right\} \\
= & o\left\{\frac{1}{\log n}\right\} \text { using (3.2) } \\
= & o(1), \text { as } n \rightarrow \infty
\end{aligned}
$$

Now,

$$
\begin{gathered}
\left|\mathrm{I}_{2}\right| \leq \int_{1 / \mathrm{n}}^{\delta}|\varnothing(\mathrm{t})|\left|\mathrm{k}_{\mathrm{n}}(\mathrm{t})\right| \mathrm{dt} \\
=0\left[\int_{1 / \mathrm{n}}^{\delta}|\varnothing(\mathrm{t})|\left(\frac{1}{\mathrm{t}}\right) \mathrm{dt}\right] \text { by lemma } 2 \\
=0\left[\left\{\frac{1}{\mathrm{t}} \varnothing(\mathrm{t})\right\}_{1 / \mathrm{n}}^{\delta}+\int_{1 / \mathrm{n}}^{\delta} \frac{1}{\mathrm{t}^{2}} \phi(\mathrm{t}) \mathrm{dt}\right] \\
=0\left[\mathrm{o}\left\{\frac{1}{\alpha\left(\frac{1}{\mathrm{t}}\right) \mathrm{p}_{\mathrm{t}}}\right\}_{1 / \mathrm{n}}^{\delta}+\int_{1 / \mathrm{n}}^{\delta} \mathrm{o}\left(\frac{1}{\mathrm{t} \alpha\left(\frac{1}{\mathrm{t}}\right) \mathrm{p}_{\mathrm{t}}}\right) \mathrm{dt}\right] \mathrm{by}(3.1) \\
=\mathrm{Putting} \frac{1}{\mathrm{t}}=\mathrm{u} \text { in second term, } \\
\left.0\left\{\frac{1}{\alpha(\mathrm{n}) \mathrm{p}_{\mathrm{n}}}\right\}+\int_{1 / \delta}^{\mathrm{n}} \mathrm{o}\left(\frac{1}{\mathrm{u} \alpha(\mathrm{u}) \mathrm{p}_{\mathrm{u}}}\right) \mathrm{du}\right] \\
=\mathrm{o}\left\{\frac{1}{\alpha(\mathrm{n}) \mathrm{p}_{\mathrm{n}}}\right\}+\mathrm{o}\left\{\frac{1}{\mathrm{n} \alpha(\mathrm{n}) \mathrm{p}_{\mathrm{n}}}\right\} \int_{1 / \delta}^{\mathrm{n}} 1 . \mathrm{du} \\
=\mathrm{o}\left\{\frac{1}{\log \mathrm{n}}\right\}+\mathrm{o}\left\{\frac{1}{\log \mathrm{n}}\right\} \mathrm{by}(3.2)
\end{gathered}
$$

Using second mean value theorem for the integral in the second term as $\alpha(n)$ is monotonic

$$
\begin{aligned}
& =o(1)+o(1) \text { as, } \mathrm{n} \rightarrow \infty \\
& =\mathrm{o}(1), \text { as } \mathrm{n} \rightarrow \infty
\end{aligned}
$$

By Rieman-Lebesgue theorem and by regularity condition of the method of Summabilty,

$$
\begin{aligned}
& \left|\mathrm{I}_{3}\right| \leq \int_{\delta}^{\pi}|\varnothing(\mathrm{t})|\left|\mathrm{k}_{\mathrm{n}}(\mathrm{t})\right| \mathrm{dt} \\
= & \mathrm{o}(1) \text {, as } \mathrm{n} \rightarrow \infty
\end{aligned}
$$

Combining (5.3), (5.4) and (5.5) we have

$$
\mathrm{E}_{\mathrm{n}}^{1} \mathrm{E}_{\mathrm{n}}^{\mathrm{q}}-\mathrm{f}(\mathrm{x})=\mathrm{o}(1) \text {, as } \mathrm{n} \rightarrow \infty
$$

This completes the proof of theorem 1 .

5.2 Proof of Theorem. Let $\widetilde{s_{n}}(f ; x)$ denotes the partial sum of series (2.2).

Then following Lal[4] and using Riemann-Lebesgue Theorem, $\widetilde{\mathrm{s}_{\mathrm{n}}}(\mathrm{f} ; \mathrm{x})$ of series (2.2) is given by

$$
\widetilde{s_{n}}(f ; x)-\tilde{f}(x)=\frac{1}{2 \pi} \int_{0}^{\pi} \emptyset(t) \frac{\cos \left(n+\frac{1}{2}\right) t}{\sin \frac{t}{2}} d t
$$

Therefore using (2.2), the (E,q) transform $E_{n}^{q}$ of $\widetilde{s_{n}}(f ; x)$ is given by 


$$
\widetilde{\mathrm{E}}_{\mathrm{n}}^{\mathrm{q}}-\tilde{\mathrm{f}}(\mathrm{x})=\frac{1}{2 \pi(1+\mathrm{q})^{\mathrm{k}}} \int_{0}^{\pi} \psi(\mathrm{t})\left\{\sum_{\mathrm{v}=0}^{\mathrm{k}}\left(\begin{array}{l}
\mathrm{k} \\
\mathrm{v}
\end{array}\right) \mathrm{q}^{\mathrm{k}-\mathrm{v}} \frac{\cos \left(\mathrm{k}+\frac{1}{2}\right) \mathrm{t}}{\sin \frac{\mathrm{t}}{2}}\right\} \mathrm{dt}
$$

Now denoting $\overline{(E, 1)(E, q)}$ transform of $\widetilde{s_{n}}(f ; x)$ by $\overline{E_{n}^{1} E_{n}^{q}}$ we write

$$
\begin{gathered}
\overline{\mathrm{E}_{\mathrm{n}}^{1} \mathrm{E}_{\mathrm{n}}^{\mathrm{q}}}-\tilde{\mathrm{f}}(\mathrm{x})=\frac{1}{2 \pi \cdot(2)^{\mathrm{n}}} \sum_{\mathrm{k}=0}^{\mathrm{n}}\left[\frac{1}{(1+\mathrm{q})^{\mathrm{k}}}\left(\begin{array}{l}
\mathrm{n} \\
\mathrm{k}
\end{array}\right) \int_{0}^{\pi} \psi(\mathrm{t})\left\{\sum_{\mathrm{v}=0}^{\mathrm{k}}\left(\begin{array}{l}
\mathrm{k} \\
\mathrm{v}
\end{array}\right) \mathrm{q}^{\mathrm{k}-\mathrm{v}} \frac{\cos \left(\mathrm{k}+\frac{1}{2}\right) \mathrm{t}}{\sin \frac{\mathrm{t}}{2}}\right\} \mathrm{dt}\right] \\
=\frac{1}{2^{\mathrm{n}+1} \cdot \pi} \sum_{\mathrm{k}=0}^{\mathrm{n}}\left[\frac{1}{(1+\mathrm{q})^{\mathrm{k}}}\left(\begin{array}{l}
\mathrm{n} \\
\mathrm{k}
\end{array}\right) \int_{0}^{\pi} \psi(\mathrm{t})\left\{\sum_{\mathrm{v}=0}^{\mathrm{k}}\left(\begin{array}{l}
\mathrm{k} \\
\mathrm{v}
\end{array}\right) \mathrm{q}^{\mathrm{k}-\mathrm{v}} \frac{\cos \left(\mathrm{k}+\frac{1}{2}\right) \mathrm{t}}{\sin \frac{\mathrm{t}}{2}}\right\} \mathrm{dt}\right] \\
=\int_{0}^{\pi} \psi(\mathrm{t}) \widetilde{\mathrm{k}_{\mathrm{n}}}(\mathrm{t}) \mathrm{dt}
\end{gathered}
$$

In order to prove the Theorem, we have to show that, under the hypothesis of theorem

$$
\int_{0}^{\pi} \psi(\mathrm{t}) \widetilde{\mathrm{k}_{\mathrm{n}}}(\mathrm{t}) \mathrm{dt}=\mathrm{o}(1) \quad \text { as } \mathrm{n} \rightarrow \infty
$$

For $0<\delta<\pi$, we have

$$
\begin{aligned}
\int_{0}^{\pi} \psi(\mathrm{t}) \widetilde{\mathrm{k}_{\mathrm{n}}}(\mathrm{t}) \mathrm{dt} & =\left[\int_{0}^{1 / \mathrm{n}} \psi(\mathrm{t})+\int_{1 / \mathrm{n}}^{\delta} \psi(\mathrm{t})+\int_{\delta}^{\pi} \psi(\mathrm{t})\right] \widetilde{\mathrm{k}_{\mathrm{n}}}(\mathrm{t}) \mathrm{dt} \\
& =\mathrm{J}_{1}+\mathrm{J}_{2}+\mathrm{J}_{3} \quad \text { (Say) }
\end{aligned}
$$

We consider,

$$
\begin{gathered}
\left|J_{1} \leq\right| \int_{0}^{1 / n}|\psi(\mathrm{t})|\left|\widehat{\mathrm{k}_{\mathrm{n}}(\mathrm{t})}\right| \mathrm{dt} \\
=\mathrm{O}\left[\int_{0}^{1 / \mathrm{n}} \frac{1}{\mathrm{t}}|\psi(\mathrm{t})| \mathrm{dt}\right] \text { by lemma } 3 \\
=O(n)\left[\int_{0}^{1 / \mathrm{n}}|\psi(\mathrm{t})| \mathrm{dt}\right] \\
=\mathrm{O}(\mathrm{n})\left[\mathrm{o}\left\{\frac{1}{\mathrm{n} \alpha(\mathrm{n}) \cdot \mathrm{p}_{\mathrm{n}}}\right\}\right] \text { by } 2.1 \\
=\mathrm{o}\left\{\frac{1}{\alpha(\mathrm{n}) \cdot \mathrm{p}_{\mathrm{n}}}\right\} \\
=\mathrm{o}\left\{\frac{1}{\log \mathrm{n}}\right\} \text { using } 2.2 \\
=\mathrm{o}(1), \text { as } \mathrm{n} \rightarrow \infty
\end{gathered}
$$

Now,

$$
\begin{aligned}
& \left|J_{2}\right| \leq \int_{1 / \mathrm{n}}^{\delta}|\psi(\mathrm{t})|\left|\widetilde{\mathrm{k}_{\mathrm{n}}}(\mathrm{t})\right| \mathrm{dt} \\
= & \mathrm{O}\left[\int_{1 / \mathrm{n}}^{\delta} \frac{1}{\mathrm{t}}|\psi(\mathrm{t})| \mathrm{dt}\right] \text { by lemma } \\
= & O\left[\left\{\frac{1}{\mathrm{t}} \psi(\mathrm{t})\right\}_{1 / \mathrm{n}}^{\delta}+\int_{1 / \mathrm{n}}^{\delta} \frac{1}{\mathrm{t}^{2}} \psi(\mathrm{t}) \mathrm{dt}\right] \\
= & 0\left[\mathrm{o}\left\{\frac{1}{\alpha\left(\frac{1}{\mathrm{t}}\right) \mathrm{p}_{\mathrm{t}}}\right\}_{1 / \mathrm{n}}^{\delta}+\int_{1 / \mathrm{n}}^{\delta} \mathrm{o}\left(\frac{1}{\mathrm{t} \alpha\left(\frac{1}{\mathrm{t}}\right) \mathrm{p}_{\mathrm{t}}}\right) \mathrm{dt}\right] \text { by }
\end{aligned}
$$

Putting $\frac{1}{\mathrm{t}}=\mathrm{u}$, in second term,

$$
=0\left[o\left\{\frac{1}{\alpha(\mathrm{n}) \mathrm{p}_{\mathrm{n}}}\right\}+\int_{1 / \delta}^{\mathrm{n}} \mathrm{o}\left(\frac{1}{\mathrm{u} \alpha(\mathrm{u}) \mathrm{p}_{\mathrm{t}}}\right) \mathrm{du}\right]
$$




$$
\begin{gathered}
=o\left\{\frac{1}{\alpha(n) p_{n}}\right\}+o\left\{\frac{1}{n \alpha(n) p_{n}}\right\} \int_{1 / \delta}^{n} 1 \cdot d u \\
=o\left\{\frac{1}{\log n}\right\}+o\left\{\frac{1}{\log n}\right\} \text { by }(2.2)
\end{gathered}
$$

Using second -mean value theorem for the integral in the second term as $\alpha(n)$ is monotonic

$$
\begin{aligned}
& =o(1)+o(1), \text { as } n \rightarrow \infty \\
& =o(1), \text { as } n \rightarrow \infty
\end{aligned}
$$

By Riemann - Lebesgue theorem and by regularity condition of the method of Summability

$$
\begin{aligned}
\left|\mathrm{J}_{3}\right| & \leq \int_{\delta}^{\pi}|\psi(\mathrm{t})|\left|\widetilde{\mathrm{k}}_{\mathrm{n}}(\mathrm{t})\right| \mathrm{dt} \\
& =\mathrm{o}(1), \text { as } \mathrm{n} \rightarrow \infty
\end{aligned}
$$

Combining (5.8), (5.9) and (5.10) we have,

$$
\overline{E_{n}^{1} E_{n}^{q}}-\tilde{f}(x)=o(1), \text { as } n \rightarrow \infty
$$

This completes the proof of theorem 2 .

\section{Conclusion}

In the field of Summability theory, various results pertaining $(\mathrm{E}, 1)$ and $(\mathrm{E}, \mathrm{q}),(\mathrm{E}, 1) \mathrm{X}$ and $\mathrm{X}(\mathrm{E}, 1)$ Summability of Fourier series as well as its allied series have been reviewed.

In future, the present work can be extended to establish new results under certain conditions.

\section{REFERENCES}

[1] Chandra, p.on the $|\mathrm{E}, \mathrm{q}|$ summability of a Fourier series and its conjugate series. Riv, Mat, Univ. Parma (4), 3, 65-78(1977).

[2] Chandra, p. and Dikshit, G.D. , on the $|B|$ and $|E, q|$ summability of a Fourier series, its conjugate series and their derived series, Indian J. pure applications math.,12(11) 1350 - 1360, (1981).

[3] Hare Krishna Nigam, kusum Sharma, on (E,1) (C,1) Summability of Fourier series and its conjugate series, Deemed university, 365 - 375, 2013

[4] H.K. Nigam, on (C,2) (E,1) product means of Fourier series and its conjugate series,1(2), 334344, 2013.

[5] G.H. Hardy, Divergent series, first edition, oxford university (1949).

[6] Nigam, H.K. and Sharma, Ajay, on (N,P,q) (E,1) Summability of Fourier series, IJMMS, vol. 2009 , (2009).

[7] Tiwari, Sandeep kumar and Bariwal chandrashekhar, degree of approximation of function belonging to the IJMA 1(1 ), 2-4, (2010).

[8] E.C. Titchmarsh, the theory of functions, oxford university press (1939), 402- 403.

\section{AUTHORS' BIOGRAPHY}

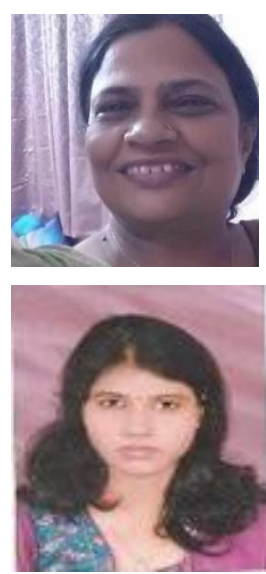

Dr. Kalpana Saxena, received Ph.D. in Mathematics from University of APS Rewa 1999. Dr. Kalpana Saxena is presently posted as a professor at GOVT. M.V.M. College Bhopal. Her main interests are Product Summability of Fourier series and sequence.

Sheela Verma, perusing Ph.D. in Mathematics from Barkatullah University under the guidance of Dr. Kalpana Saxena. I completed Msc. In mathematics From Sarojini naidu GOVT. Girl's P.G. College Shivaji Nagar Bhopal. 